\title{
Identification of Hammerstein Systems Using Separable Random Multisines
}

\author{
Martin Enqvist \\ Division of Automatic Control \\ Department of Electrical Engineering \\ Linköpings universitet, SE-581 83 Linköping, Sweden \\ WWW: http://wWw. control.isy.liu.se \\ E-mail: maren@isy.liu.se
}

30th December 2005

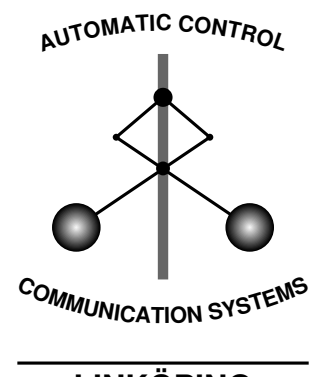

Report no.: LiTH-ISY-R-2722

Submitted to the 14th IFAC Symposium on System Identification,

Newcastle, Australia

Technical reports from the Control \& Communication group in Linköping are available at http://www.control.isy.liu.se/publications. 


\begin{abstract}
The choice of input signal is very important in identification of nonlinear systems. In this paper, it is shown that random multisines with a flat amplitude spectrum are separable. The separability property means that certain conditional expectations are linear and it implies that random multisines easily can be used to obtain accurate estimates of the linear time-invariant part of a Hammerstein system. This is illustrated in a numerical example.
\end{abstract}

Keywords: System identification, Random multisines, Separable processes, Hammerstein systems 


\title{
Identification of Hammerstein Systems Using Separable Random Multisines
}

\author{
Martin Enqvist
}

2005-12-30

\begin{abstract}
The choice of input signal is very important in identification of nonlinear systems. In this paper, it is shown that random multisines with a flat amplitude spectrum are separable. The separability property means that certain conditional expectations are linear and it implies that random multisines easily can be used to obtain accurate estimates of the linear time-invariant part of a Hammerstein system. This is illustrated in a numerical example.
\end{abstract}

\section{Introduction}

A Hammerstein system consists of a static nonlinearity followed by a linear time-invariant (LTI) system. This system structure is common in many real-life applications and it is thus natural that identification of Hammerstein systems has been an active research field for quite some time, see, for example, Narendra and Gallman (1966); Chang and Luus (1971); Stoica (1981); Billings and Fakhouri $(1978,1982)$. A brief overview of some of the existing methods can be found in Bai and $\mathrm{Li}$ (2004).

A parameterized Hammerstein model can be written as

$$
y(t)=G(q, \theta) f(u(t), \eta)+e(t),
$$

where $q$ is the shift operator $q u(t)=u(t+1)$, where $u(t)$ and $y(t)$ are the input and output signals, respectively, and where $e(t)$ is measurement noise. Here, $\theta$ and $\eta$ are the parameters of the linear and nonlinear subsystem, respectively. If $N$ input and output measurements are collected from a particular Hammerstein system, the parameters $\theta$ and $\eta$ can be found by minimizing a cost function, for example a quadratic function

$$
V_{N}(\theta, \eta)=\frac{1}{N} \sum_{t=0}^{N-1}(y(t)-G(q, \theta) f(u(t), \eta))^{2} .
$$

In many cases, it is hard to simultaneously minimize $V_{N}(\theta, \eta)$ with respect to $\theta$ and $\eta$. Instead, a couple of iterative methods have been suggested.

One popular method for identification of Hammerstein systems was proposed in Narendra and Gallman (1966). In this method, the minimization of $V_{N}(\theta, \eta)$ is done iteratively starting from an initial estimate, or guess, of the parameter 
values. In each step, the function $V_{N}(\theta, \eta)$ is minimized with respect only to one of its arguments while using the previous value of the other one. Often, an LTI approximation of the system is first estimated without considering the nonlinearity at the input and the parameters from this approximate model are used as $\theta$-parameters when the first estimate of $\eta$ is calculated in the next step. For example, the initial LTI model can be obtained by using the prediction-error method (Ljung, 1999). With an output error (OE) model structure, this means that the parameter estimate is calculated by minimizing the function

$$
\frac{1}{N} \sum_{t=0}^{N-1}(y(t)-G(q, \theta) u(t))^{2}
$$

numerically with respect to $\theta$.

The iterative approach guarantees that the cost function will be monotonically decreasing over the iterations. Furthermore, the convergence of the parameter estimates can be shown in some special cases (Bai and Li, 2004). However, there are no convergence results available for the general case. Hence, it is important that the initial LTI approximation is as good as possible in the sense that it resembles the true LTI subsystem as well as possible.

For a Gaussian input signal, the iterative approach can be motivated by Bussgang's theorem (Bussgang, 1952). This result implies that for a Hammerstein system, the LTI model that minimizes the mean-square error

$$
\mathrm{E}\left((y(t)-G(q) u(t))^{2}\right)
$$

will be equal to a scaled version of the LTI part of the system. Since the cost function (3) in most cases will be a good approximation of the meansquare error (4) for large $N$, Bussgang's theorem guarantees that an OE model estimated from a large data set using the prediction-error method will be a good approximation of the LTI subsystem. Bussgang's theorem has been extended also to signals that are separable in Nuttall's sense (see Definition 2.3) (Nuttall, 1958a,b; McGraw and Wagner, 1968). Identification of Hammerstein systems using separable input signals has been discussed in, for example, Billings and Fakhouri (1978). The theory for separable processes has also been extended to systems where the nonlinear subsystem is a nonlinear finite impulse response (NFIR) system (Enqvist and Ljung, 2005). Related material can be found in Enqvist (2005).

For nonseparable input signals, the estimation of the initial LTI model requires more attention. A number of results concerning this problem for random multisine inputs can be found in Crama and Schoukens (2001); Crama et al. (2004); Crama and Schoukens (2004, 2005). General theory about random multisines and linear approximations of nonlinear systems can, for example, be found in Pintelon and Schoukens (2001); Schoukens et al. (2005).

The main contribution of this paper is the observation that random multisines with a flat amplitude spectrum are separable. This result provides a theoretical explanation to why previous methods for identification of Hammerstein systems using random multisines have been so successful. 


\section{Preliminaries}

In this section, some background theory about LTI approximations of nonlinear systems and separable processes is presented.

\subsection{Second Order Equivalents}

In this paper, we will study nonlinear systems with periodic inputs. More specifically, the signal assumptions are as follows.

Assumption A1. Assume that the input $u(t)$ and output $y(t)$ are real-valued stationary stochastic processes with $\mathrm{E}(u(t))=\mathrm{E}(y(t))=0$ and that $u(t)$ is $P$-periodic for some $P \in \mathbb{Z}_{+}$, i.e., that

$$
u(t+P)=u(t), \quad \forall t \in \mathbb{Z} .
$$

Furthermore, assume that the covariance functions $R_{u}(\tau)=\mathrm{E}(u(t) u(t-\tau))$ and $R_{y u}(\tau)=\mathrm{E}(y(t) u(t-\tau))$ exist and are $P$-periodic.

Approximations of nonlinear systems can be derived using different theoretical frameworks. In this paper, LTI models that are optimal approximations in the mean-square error sense are studied. Such a model will here be called an Output Error LTI Second Order Equivalent (OE-LTI-SOE), and it is defined in the following definition.

Definition 2.1. Consider a nonlinear system with a $P$-periodic input $u(t)$ and an output $y(t)$ such that Assumption A1 is fulfilled. An OE-LTI-SOE of this system is a stable and causal LTI model $G_{0, O E}(q)$ that minimizes the meansquare error $\mathrm{E}\left((y(t)-G(q) u(t))^{2}\right)$, i.e.,

$$
G_{0, O E}(q)=\underset{G \in \mathcal{G}}{\arg \min } \mathrm{E}\left((y(t)-G(q) u(t))^{2}\right),
$$

where $\mathcal{G}$ denotes the set of all stable and causal LTI models. Let $\mathcal{G}_{0, O E}$ denote the set of all OE-LTI-SOEs for this particular pair of input and output signals, i.e.,

$$
\mathcal{G}_{0, O E}=\left\{G_{0, O E}(q)\right\} .
$$

Note that $\mathcal{G}_{0, O E}$ always will contain more than one model. For example, consider a system with an OE-LTI-SOE $G_{0, O E, 0}(q)$ for a particular $P$-periodic input $u(t)$. Then the models $G_{0, O E, k}(q)=\left(1+q^{-k P}\right) G_{0, O E, 0}(q) / 2, k \in \mathbb{N}$, are OE-LTI-SOEs too, since they will produce the same stationary output as $G_{0, O E, 0}(q)$. Obviously, the impulse responses from these OE-LTI-SOEs are quite different, but this does not matter here since the transient response of a model is not considered in the definition of the OE-LTI-SOE.

Usually, the mean-square error cannot be minimized directly in real identification problems. Instead, with data sets from $N_{E}$ experiments where different realizations of the input signal have been used and $N=M P, M \in \mathbb{Z}_{+}$measurements in each data set, a model can be estimated by minimizing the approximate cost function

$$
V_{N_{E}, N}(G(q))=\frac{1}{N_{E}} \sum_{s=1}^{N_{E}} \frac{1}{N} \sum_{t=0}^{N-1}\left(y_{s}(t)-G(q) u_{s}(t)\right)^{2}
$$


with respect to $G(q)$. Here, $u_{s}(t)$ and $y_{s}(t)$ are the input and output signals from experiment $s$, respectively.

With a periodic input it is very natural to consider the modeling problem in the frequency domain. Applying the Discrete Fourier Transform (DFT) to the input and output signals gives the transforms

$$
\begin{aligned}
& U_{s, N}(n)=\sum_{t=0}^{N-1} u_{s}(t) e^{-i 2 \pi n t / N}, \\
& Y_{s, N}(n)=\sum_{t=0}^{N-1} y_{s}(t) e^{-i 2 \pi n t / N} .
\end{aligned}
$$

Let $\hat{y}_{G, s}(t)$ denote the output from the stable model $G(q)$ for the input $u_{s}(t)$ and assume that the input has been applied at $t=-\infty$ such that all transients have disappeared at $t \geq 0$, i.e., that $\hat{y}_{G, s}(t)$ is $P$-periodic in the interval $0 \leq t \leq N-1$. Furthermore, let $\hat{Y}_{G, s, N}(n)$ denote the DFT of $\hat{y}_{G, s}(t)$, i.e.,

$$
\hat{Y}_{G, s, N}(n)=\sum_{t=0}^{N-1} \hat{y}_{G, s}(t) e^{-i 2 \pi n t / N} .
$$

The frequency response of the stable model $G(z)$ is obtained for $z=e^{i \omega}$. In particular, since $r(t)=e^{-i 2 \pi n t / N}$ is an $N$-periodic signal, it follows that

$$
\begin{aligned}
G\left(e^{i 2 \pi n / N}\right) & =\sum_{k=0}^{\infty} g(k) e^{-i 2 \pi n k / N} \\
& =\sum_{t=0}^{N-1} \underbrace{\left(\sum_{l=0}^{\infty} g(t+l N)\right)}_{=: \tilde{g}_{N}(t)} e^{-i 2 \pi n t / N}=: \tilde{G}_{N}(n) .
\end{aligned}
$$

Furthermore, since $u_{s}(t)$ is a $P$-periodic signal and $N=M P, M \in \mathbb{Z}_{+}, u_{s}(t)$ is also $N$-periodic. Hence,

$$
\hat{y}_{G, s}(t)=G(q) u_{s}(t)=\sum_{k=0}^{N-1} \tilde{g}(k) u_{s}(t-k)
$$

and this implies that

$$
\hat{Y}_{G, s, N}(n)=\tilde{G}_{N}(n) U_{s, N}(n)=G\left(e^{i 2 \pi n / N}\right) U_{s, N}(n),
$$

where we have used (6) in the last equality.

Using Parseval's formula, the cost function can be rewritten as

$$
\begin{aligned}
V_{N_{E}, N}(G(q)) & =\frac{1}{N_{E}} \sum_{s=1}^{N_{E}} \frac{1}{N} \sum_{t=0}^{N-1}(y_{s}(t)-\underbrace{G(q) u_{s}(t)}_{=\hat{y}_{G, s}(t)})^{2} \\
& =\frac{1}{N_{E} N^{2}} \sum_{s=1}^{N_{E}} \sum_{n=0}^{N-1}\left|Y_{s, N}(n)-\hat{Y}_{G, s, N}(n)\right|^{2} \\
& =\frac{1}{N_{E} N^{2}} \sum_{s=1}^{N_{E}} \sum_{n=0}^{N-1}\left|Y_{s, N}(n)-G\left(e^{i 2 \pi n / N}\right) U_{s, N}(n)\right|^{2} .
\end{aligned}
$$


From this expression, it is obvious that two linear models will give the same value of the cost function if their frequency responses are equal at the frequencies where $U_{s, N}(n)$ is nonzero. In particular, if an optimal nonparametric frequency response estimate is calculated by minimizing $V_{N_{E}, N}(G(q))$ for each excited frequency, any other optimal model must have a frequency response that is equal to the optimal nonparametric one at these frequencies and that can have arbitrary values at all other frequencies. Hence, all information about the set of optimal models can be found by calculating the optimal nonparametric model at the excited frequencies. This observation will be used later when a particular Hammerstein system is studied in a numerical example.

\subsection{Random Multisines}

In this paper, only one type of periodic input signals will be considered, namely random multisines.

Definition 2.2. A random multisine signal is a stationary stochastic process $u(t)$ that can be written

$$
u(t)=\sum_{k=1}^{Q} A_{k} \cos \left(\omega_{k} t+\psi_{k}\right),
$$

where both $A_{k}$ and $\psi_{k}$ can be random variables and where all $\omega_{k}$ are constants that satisfy $\left|\omega_{k}\right| \leq \pi$.

Here, the phases $\psi_{k}$ will usually be independent random variables with uniform distribution on the interval $[0,2 \pi)$ and the amplitudes $A_{k}$ will usually be constants. Furthermore, we will only consider periodic random multisines such that the period $P$ is an integer, i.e., such that all $\omega_{k}$ can be written $\omega_{k}=\pi p_{k}$ for some $p_{k} \in\{x \in \mathbb{Q}|| x \mid \leq 1\}$.

\subsection{Separable Processes}

The results in this paper concern separable processes, i.e., processes that satisfy the condition described in the following definition.

Definition 2.3 (Separability). A stationary stochastic process $u(t)$ with $\mathrm{E}(u(t))=0$ is separable (in Nuttall's sense) if

$$
\mathrm{E}(u(t-\tau) \mid u(t))=a(\tau) u(t) .
$$

for some function $a(\tau)$.

It is easy to show that the function $a(\tau)$ in $(9)$ can be expressed using the covariance function of $u(t)$.

Lemma 2.1

Consider a separable stationary stochastic process $u(t)$ with $\mathrm{E}(u(t))=0$. The function $a(\tau)$ from $(9)$ can then be written

$$
a(\tau)=\frac{R_{u}(\tau)}{R_{u}(0)}
$$


Proof: Nuttall (1958a)

Some properties of a separable stochastic process can be expressed also using the characteristic function of the process.

Definition 2.4. Consider a stationary stochastic process $u(t)$ with $\mathrm{E}(u(t))=0$. Let the first order characteristic function be denoted with

$$
f_{u, 1}\left(\xi_{1}\right)=\mathrm{E}\left(e^{i \xi_{1} u(t)}\right) .
$$

A single sinusoid with random phase is separable according to the following lemma.

\section{Lemma 2.2}

A random sine process

$$
u(t)=A \cos (\omega t+\psi),
$$

where $\psi$ is a random variable with uniform distribution on the interval $[0,2 \pi)$ and where $A$ and $\omega$ are constants, is a separable process. Furthermore, this process has the properties

$$
\begin{aligned}
R_{u}(\tau) & =\frac{A^{2}}{2} \cos (\omega \tau), \\
f_{u, 1}\left(\xi_{1}\right) & =J_{0}\left(A \xi_{1}\right),
\end{aligned}
$$

where $J_{0}$ is the zeroth order Bessel function.

Proof: Nuttall (1958a)

In the next theorem, it is shown that the sum of $Q$ independent separable processes is separable if the characteristic functions satisfy a certain condition.

\section{Theorem 2.1}

Consider $Q$ independent and separable stationary stochastic processes $u_{k}(t)$ with

$$
\mathrm{E}\left(u_{k}(t)\right)=0
$$

for $k=1, \ldots, Q$ and let

$$
u(t)=\sum_{k=1}^{Q} u_{k}(t) .
$$

Assume that the characteristic functions satisfy

$$
f_{u_{k}, 1}\left(\xi_{1}\right)^{1 / \sigma_{k}^{2}}=f_{u_{l}, 1}\left(\xi_{1}\right)^{1 / \sigma_{l}^{2}},
$$

for all $k, l \in\{1,2, \ldots, Q\}$, where $\sigma_{m}^{2}=R_{u_{m}}(0)$. Then $u(t)$ is separable.

Proof: Nuttall (1958a)

In the next section, Theorem 2.1 will be used to show that some random multisines are separable. 


\section{Separable Random Multisines}

The main result in this paper concerns the separability of random multisines. It turns out that such signals are separable if all amplitudes are constant and equal. This result is proven in the following lemma.

Lemma 3.1

$A$ random multisine

$$
u(t)=\sum_{k=1}^{Q} A_{k} \cos \left(\omega_{k} t+\psi_{k}\right),
$$

where all $A_{k}$ are constants, $A_{k}=\bar{A}$, and all $\psi_{k}$ are independent random variables with uniform distribution on the interval $[0,2 \pi)$, is separable.

Proof: The signals

$$
u_{k}(t)=A_{k} \cos \left(\omega_{k} t+\psi_{k}\right)
$$

are independent and

$$
\begin{aligned}
f_{u_{k}, 1}\left(\xi_{1}\right) & =f_{u_{l}, 1}\left(\xi_{1}\right)=J_{0}\left(\bar{A} \xi_{1}\right), \\
\sigma_{k}^{2} & =\sigma_{l}^{2}=\frac{\bar{A}^{2}}{2}
\end{aligned}
$$

for all $k, l \in\{1,2, \ldots, Q\}$ from Lemma 2.2. Hence, $u(t)$ is separable according to Theorem 2.1 .

Besides the fact that the separability of random multisines is theoretically interesting, it has some practical implications for identification of Hammerstein systems. This will be discussed in the next section.

\section{Hammerstein Systems}

As has been mentioned previously, the initial identification of the LTI part of a Hammerstein system without considering the nonlinearity is an important step in many methods. In the following theorem, it is shown that a scaled version of the LTI subsystem is an OE-LTI-SOE of the system if the input is a separable random multisine. Hence, the model that minimize $V_{N_{E}, N}$ will usually be a good approximation of the LTI subsystem if $N_{E}$ is large.

\section{Theorem 4.1}

Consider a Hammerstein system

$$
\begin{aligned}
& y(t)=G_{L}(q) v(t)+w(t), \\
& v(t)=f(u(t)),
\end{aligned}
$$

where $G_{L}(q)$ is a stable and causal LTI system and where $w(t)$ is measurement noise with $\mathrm{E}(w(t))=0$. Assume that the input to this system is a P-periodic random multisine, $P \in \mathbb{Z}_{+}$,

$$
u(t)=\sum_{k=1}^{Q} A_{k} \cos \left(\omega_{k} t+\psi_{k}\right),
$$


where all $A_{k}$ are constants, $A_{k}=\bar{A}$, and all $\psi_{k}$ are independent random variables with uniform distribution on the interval $[0,2 \pi)$. Assume that $u(t)$ and $w(s)$ are independent for all $t, s \in \mathbb{Z}$ and that Assumption A1 holds. Then

$$
c_{0} G_{L}(q) \in \mathcal{G}_{0, O E},
$$

where $c_{0}=\mathrm{E}(f(u(t)) u(t)) / R_{u}(0)$ is a constant.

Proof: The equalities (9) and (10) hold since $u(t)$ by Lemma 3.1 is separable. Hence,

$$
\begin{aligned}
R_{v u}(\tau) & =\mathrm{E}(f(u(t)) u(t-\tau)) \\
& =\mathrm{E}(f(u(t)) \mathrm{E}(u(t-\tau) \mid u(t))) \\
& =\frac{R_{u}(\tau)}{R_{u}(0)} \mathrm{E}(f(u(t)) u(t))=c_{0} R_{u}(\tau) .
\end{aligned}
$$

The condition that $G_{0, O E}$ should minimize

$$
\mathrm{E}\left((y(t)-G(q) u(t))^{2}\right)
$$

is equivalent to $G_{0, O E}$ satisfying the Wiener-Hopf condition

$$
R_{y u}(\tau)-\sum_{k=0}^{\infty} g_{0, O E}(k) R_{u}(\tau-k)=0,0 \leq \tau \leq P-1 .
$$

From the system description (16a), we have

$$
R_{y u}(\tau)-\sum_{k=0}^{\infty} g_{L}(k) R_{v u}(\tau-k)=0, \quad \forall \tau \in \mathbb{Z}
$$

and inserting (18) gives

$$
R_{y u}(\tau)-\sum_{k=0}^{\infty} c_{0} g_{L}(k) R_{u}(\tau-k)=0, \quad \forall \tau \in \mathbb{Z} .
$$

Hence, $c_{0} G_{L}(q) \in \mathcal{G}_{0, O E}$.

The previous theorem indicates that random multisines with flat amplitude spectra are suitable for identification of Hammerstein systems. In particular, it shows that the number of excited frequencies does not affect the fact that the LTI subsystem can be estimated consistently without considering the nonlinearity. Theorem 4.1 is verified numerically in the following example.

Example 4.1

Consider the Hammerstein system

$$
\begin{aligned}
& y(t)=G_{L}(q) v(t)=\frac{1.6-1.6 q^{-1}+0.4 q^{-2}}{1-1.56 q^{-1}+0.96 q^{-2}} v(t), \\
& v(t)=f(u(t))=u(t)^{3}
\end{aligned}
$$

with the input

$$
u_{1}(t)=\sum_{k=1}^{6} \cos \left(\omega_{k} t+\psi_{k}\right)
$$



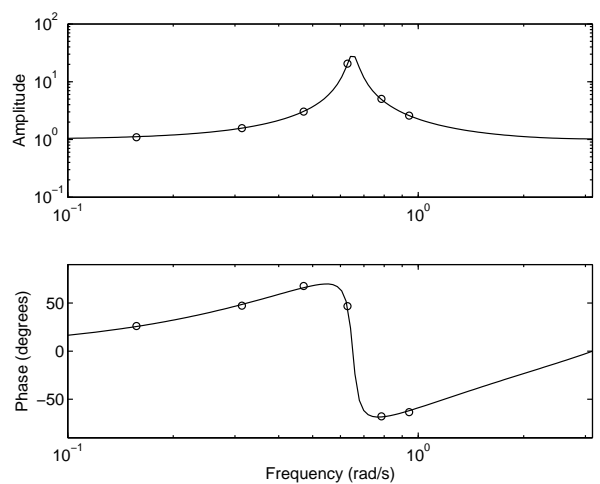

Figure 1: The frequency response of the linear part $G_{L}(q)$ of the Hammerstein system from Example 4.1 (solid line) and a scaled version of the nonparametric frequency response estimate (circles).

where $\omega_{k}=2 \pi k / 40$ and where $\psi_{k}$ are independent random variables with uniform distribution on the interval $[0,2 \pi)$. 500 realizations of the phases have been generated and an input signal with 400 samples has been constructed for each realization. For each input, an identification experiment has been performed where the last periods (40 samples) of the input and output signals have been collected.

Based on the 500 data sets, each consisting 40 input and output measurements, a nonparametric frequency response estimate $\hat{G}\left(e^{i \omega_{k}}\right)$ has been calculated using the least-squares method and the previously defined cost function $V_{N_{E}, N}$. A scaled version of this estimate is shown in Figure 1 together with the linear part of the system. As can be seen in this figure, the nonparametric frequency response estimate is very close to being a scaled version of $G_{L}\left(e^{i \omega}\right)$. Actually, the relative errors

$$
\rho_{k}=\frac{\left|\hat{G}\left(e^{i \omega_{k}}\right) / \hat{c}_{0}-G_{L}\left(e^{i \omega_{k}}\right)\right|}{\left|G_{L}\left(e^{i \omega_{k}}\right)\right|}, \quad k=1,2, \ldots, 6,
$$

where

$$
\hat{c}_{0}=\frac{1}{6} \sum_{k=1}^{6} \frac{\left|\hat{G}\left(e^{i \omega_{k}}\right)\right|}{\left|G_{L}\left(e^{i \omega_{k}}\right)\right|},
$$

are less than $4 \%$ here. An identical identification experiment has been performed with the input

$$
u_{2}(t)=\sum_{k=1}^{6} 2^{5-k} \cos \left(\omega_{k} t+\psi_{k}\right) .
$$

However, in this case the relative errors were $27 \%, 5 \%, 12 \%, 12 \%, 12 \%$ and $17 \%$, respectively, i.e., significantly larger than for the separable random multisine. Furthermore, for $u_{2}(t)$, the relative errors do not decrease if the number of phase realizations is increased. Hence, it seems that for this input, the best LTI approximation is not a scaled version of $G_{L}(q)$. 
Example 4.1 illustrates the main benefit of using separable random multisines for Hammerstein system identification since it shows that for such an input signal, it is rather easy to obtain good estimates of the LTI part of the system. Furthermore, the example indicates that not all random multisines are separable.

\section{Conclusions}

In this paper, it has been shown that random multisines with constant, flat amplitude spectra and uniformly distributed phases are separable. This implies that a scaled version of the LTI part of a Hammerstein system is an OE-LTISOE of the system and that it is rather easy to compute a consistent estimate of the LTI subsystem. The results have been verified numerically in an example.

\section{References}

E.-W. Bai and D. Li. Convergence of the iterative Hammerstein system identification algorithm. IEEE Transactions on Automatic Control, 49(11):1929 1940, 2004.

S. A. Billings and S. Y. Fakhouri. Theory of separable processes with applications to the identification of nonlinear systems. Proceedings of the IEE, 125 (9):1051-1058, 1978.

S. A. Billings and S. Y. Fakhouri. Identification of systems containing linear dynamic and static nonlinear elements. Automatica, 18(1):15-26, 1982.

J. J. Bussgang. Crosscorrelation functions of amplitude-distorted Gaussian signals. Technical Report 216, MIT Research Laboratory of Electronics, Cambridge, Massachusetts, 1952.

F. H. I. Chang and R. Luus. A noniterative method for identification using Hammerstein model. IEEE Transactions on Automatic Control, 16(5):464468, 1971.

P. Crama and J. Schoukens. Initial estimates of Wiener and Hammerstein systems using multisine excitation. IEEE Transactions on Instrumentation and Measurement, 50(6):1791-1795, 2001.

P. Crama and J. Schoukens. Hammerstein-Wiener system estimator initialization. Automatica, 40(9):1543-1550, 2004.

P. Crama and J. Schoukens. Computing an initial estimate of a WienerHammerstein system with a random phase multisine excitation. IEEE Transactions on Instrumentation and Measurement, 54(1):117-122, 2005.

P. Crama, J. Schoukens, and R. Pintelon. Generation of enhanced initial estimates for Hammerstein systems. Automatica, 40(7):1269-1273, 2004.

M. Enqvist. Linear Models of Nonlinear Systems. PhD thesis, Linköpings universitet, Linköping, Sweden, 2005. 
M. Enqvist and L. Ljung. Linear approximations of nonlinear FIR systems for separable input processes. Automatica, 41(3):459-473, 2005.

L. Ljung. System Identification: Theory for the User. Prentice Hall, Upper Saddle River, New Jersey, second edition, 1999.

D. K. McGraw and J. F. Wagner. Elliptically symmetric distributions. IEEE Transactions on Information Theory, 14(1):110-120, 1968.

K. S. Narendra and P. G. Gallman. An iterative method for the identification of nonlinear systems using a Hammerstein model. IEEE Transactions on Automatic Control, 11(3):546-550, 1966.

A. H. Nuttall. Theory and application of the separable class of random processes. Technical Report 343, MIT Research Laboratory of Electronics, Cambridge, Massachusetts, 1958a.

A. H. Nuttall. Theory and Application of the Separable Class of Random Processes. PhD thesis, MIT, Cambridge, Massachusetts, 1958b.

R. Pintelon and J. Schoukens. System Identification: A Frequency Domain Approach. IEEE Press, New York, 2001.

J. Schoukens, R. Pintelon, T. Dobrowiecki, and Y. Rolain. Identification of linear systems with nonlinear distortions. Automatica, 41(3):491-504, 2005.

P. Stoica. On the convergence of an iterative algorithm used for Hammerstein system identification. IEEE Transactions on Automatic Control, 26(4):967969, 1981. 


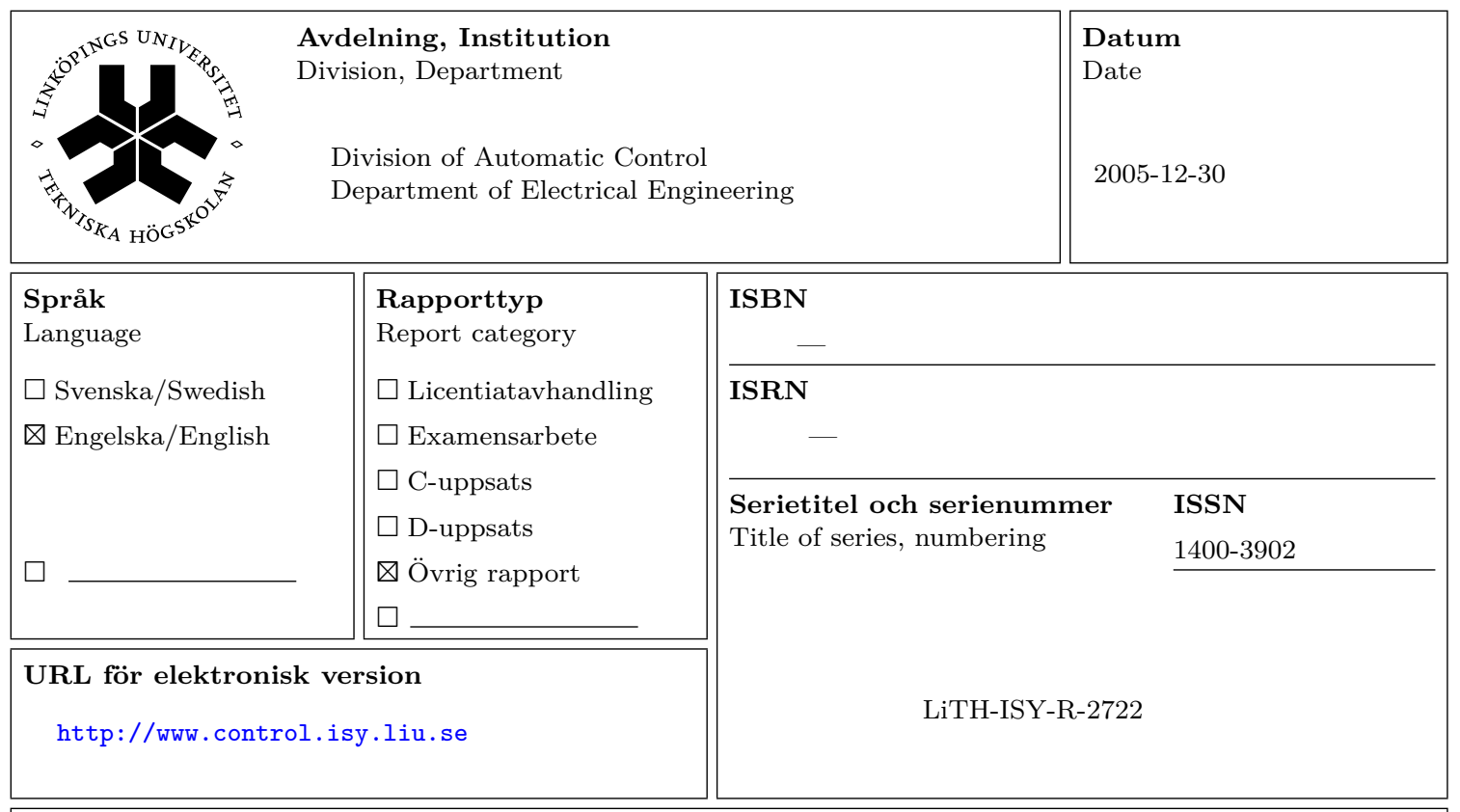

Titel Identification of Hammerstein Systems Using Separable Random Multisines

Title

Författare Martin Enqvist

Author

\section{Sammanfattning}

Abstract

The choice of input signal is very important in identification of nonlinear systems. In this paper, it is shown that random multisines with a flat amplitude spectrum are separable. The separability property means that certain conditional expectations are linear and it implies that random multisines easily can be used to obtain accurate estimates of the linear timeinvariant part of a Hammerstein system. This is illustrated in a numerical example. 